\title{
Ratio Monotonicity of Polynomials Derived from Nondecreasing Sequences
}

\author{
William Y. C. Chen ${ }^{1}$, Arthur L. B. Yang ${ }^{2}$, Elaine L. F. Zhou ${ }^{3}$ \\ Center for Combinatorics, LPMC-TJKLC \\ Nankai University, Tianjin 300071, P. R. China \\ ${ }^{1}$ chen@nankai.edu.cn, ${ }^{2}$ yang@nankai.edu.cn, ${ }^{3}$ zhoulf@cf c.nankai.edu.cn \\ Submitted: July 28, 2010; Accepted: Nov 26, 2010; Published: Dec 10, 2010 \\ Mathematics Subject Classification: 05A20, 33F10
}

\begin{abstract}
The ratio monotonicity of a polynomial is a stronger property than log-concavity. Let $P(x)$ be a polynomial with nonnegative and nondecreasing coefficients. We prove the ratio monotone property of $P(x+1)$, which leads to the log-concavity of $P(x+c)$ for any $c \geq 1$ due to Llamas and Martínez-Bernal. As a consequence, we obtain the ratio monotonicity of the Boros-Moll polynomials obtained by Chen and Xia without resorting to the recurrence relations of the coefficients.
\end{abstract}

Keywords: log-concavity, ratio monotonicity, Boros-Moll polynomials.

\section{Introduction}

This paper is concerned with the ratio monotone property of polynomials derived from nonnegative and nondecreasing sequences. A sequence $\left\{a_{k}\right\}_{0 \leq k \leq m}$ of positive real numbers is said to be unimodal if there exists an integer $r \geq 0$ such that

$$
a_{0} \leq \cdots \leq a_{r-1} \leq a_{r} \geq a_{r+1} \geq \cdots \geq a_{m},
$$

and it is said to be spiral if

$$
a_{m} \leq a_{0} \leq a_{m-1} \leq a_{1} \leq \cdots \leq a_{\left[\frac{m}{2}\right]},
$$

where $\left[\frac{m}{2}\right]$ stands for the largest integer not exceeding $\frac{m}{2}$. We say that a sequence $\left\{a_{k}\right\}_{0 \leq k \leq m}$ is log-concave if for any $1 \leq k \leq m-1$,

$$
a_{k}^{2}-a_{k+1} a_{k-1} \geq 0
$$


or equivalently,

$$
\frac{a_{0}}{a_{1}} \leq \frac{a_{1}}{a_{2}} \leq \cdots \leq \frac{a_{m-1}}{a_{m}}
$$

It is easy to see that either log-concavity or the spiral property implies unimodality, while a log-concave sequence is not necessarily spiral, and vice versa.

A stronger property, which implies both log-concavity and the spiral property, was introduced by Chen and Xia [6] and is called the ratio monotonicity. A sequence of positive real numbers $\left\{a_{k}\right\}_{0 \leq k \leq m}$ is said to be ratio monotone if

$$
\frac{a_{m}}{a_{0}} \leq \frac{a_{m-1}}{a_{1}} \leq \cdots \leq \frac{a_{m-i}}{a_{i}} \leq \cdots \leq \frac{a_{m-\left[\frac{m-1}{2}\right]}}{a_{\left[\frac{m-1}{2}\right]}} \leq 1
$$

and

$$
\frac{a_{0}}{a_{m-1}} \leq \frac{a_{1}}{a_{m-2}} \leq \cdots \leq \frac{a_{i-1}}{a_{m-i}} \leq \cdots \leq \frac{a_{\left[\frac{m}{2}\right]-1}}{a_{m-\left[\frac{m}{2}\right]}} \leq 1 .
$$

Given a polynomial $P(x)=a_{0}+a_{1} x+\cdots+a_{m} x^{m}$ with positive coefficients, we say that $P(x)$ is log-concave (or ratio monotone) if $\left\{a_{k}\right\}_{0 \leq k \leq m}$ is log-concave (resp., ratio monotone).

Assume that $P(x)$ is a polynomial with nonnegative and nondecreasing coefficients. Boros and Moll [3] proved the unimodality of $P(x+1)$ which implies the unimodality of the Boros-Moll polynomials. They posed the conjecture that the Boros-Moll polynomials are log-concave, which was confirmed by Kauers and Paule [8]. Alvarez et al. [1] showed that $P(x+n)$ is also unimodal for any positive integer $n$. Wang and Yeh [12] obtained a stronger result that $P(x+c)$ is unimodal for $c>0$. Llamas and Martínez-Bernal [9] proved that $P(x+c)$ is log-concave for $c \geq 1$.

In this paper, we prove that if $P(x)$ is a polynomial with nonnegative and nondecreasing coefficients, then $P(x+1)$ is ratio monotone. This property implies the log-concavity of $P(x+1)$. Note that by a criterion for log-concavity due to Brenti [5], the log-concavity of $P(x+1)$ leads to the log-concavity of $P(x+c)$ for $c \geq 1$, as established by Llamas and Martínez-Bernal [9]. The ratio monotonicity of $P(x+1)$ serves as a simple proof of the ratio monotonicity of the Boros-Moll polynomials obtained by Chen and Xia [7] without resorting to the recurrence relations of the coefficients.

\section{The ratio monotone property}

The main result of this paper is given below.

Theorem 2.1 If $P(x)$ is a polynomial with nonnegative and nondecreasing coefficients, then $P(x+1)$ is ratio monotone.

To prove Theorem 2.1, we need three lemmas. The first lemma is a special case of $[6$, Lemma 2.1]. 
Lemma 2.2 Suppose that $a, b, c, d, e, f$ are positive real numbers satisfying

$$
\frac{a}{b} \leq \frac{c}{d} \leq \frac{e}{f}
$$

Then

$$
\frac{a+c}{b+d} \leq \frac{e+c}{f+d}
$$

Lemma 2.3 If $B(x)$ is a ratio monotone polynomial, so is $(x+1) B(x)$.

Proof. Let

$$
B(x)=\sum_{k=0}^{m} a_{k} x^{k} \quad \text { and } \quad(x+1) B(x)=\sum_{k=0}^{m+1} b_{k} x^{k} .
$$

For each $k$ we have $b_{k}=a_{k-1}+a_{k}$, where $a_{-1}$ and $a_{m+1}$ are set to 0 .

When $m=2 n$, the ratio monotonicity of $B(x)$ states that

$$
\frac{a_{2 n}}{a_{0}} \leq \frac{a_{2 n-1}}{a_{1}} \leq \cdots \leq \frac{a_{2 n-i}}{a_{i}} \leq \cdots \leq \frac{a_{n+1}}{a_{n-1}} \leq 1
$$

and

$$
\frac{a_{0}}{a_{2 n-1}} \leq \frac{a_{1}}{a_{2 n-2}} \leq \cdots \leq \frac{a_{i-1}}{a_{2 n-i}} \leq \cdots \leq \frac{a_{n-1}}{a_{n}} \leq 1 .
$$

In order to show that $(x+1) B(x)$ is ratio monotone, we need to verify that

$$
\frac{b_{2 n+1}}{b_{0}} \leq \frac{b_{2 n}}{b_{1}} \leq \cdots \leq \frac{b_{2 n+1-i}}{b_{i}} \leq \cdots \leq \frac{b_{n+1}}{b_{n}} \leq 1
$$

and

$$
\frac{b_{0}}{b_{2 n}} \leq \frac{b_{1}}{b_{2 n-1}} \leq \cdots \leq \frac{b_{i}}{b_{2 n-i}} \leq \cdots \leq \frac{b_{n-1}}{b_{n+1}} \leq 1 .
$$

We first consider (2.3). Since

$$
\frac{a_{2 n}}{a_{0}} \leq \frac{a_{2 n-1}}{a_{1}}
$$

we see that

$$
\frac{a_{2 n}}{a_{0}} \leq \frac{a_{2 n-1}+a_{2 n}}{a_{1}+a_{0}}
$$

that is,

$$
\frac{b_{2 n+1}}{b_{0}} \leq \frac{b_{2 n}}{b_{1}}
$$

For $1 \leq i \leq n-1$, from (2.1) we deduce that

$$
\frac{a_{2 n+1-i}}{a_{i-1}} \leq \frac{a_{2 n-i}}{a_{i}} \leq \frac{a_{2 n-i-1}}{a_{i+1}} .
$$

By Lemma 2.2, we obtain

$$
\frac{a_{2 n+1-i}+a_{2 n-i}}{a_{i}+a_{i-1}} \leq \frac{a_{2 n-i}+a_{2 n-i-1}}{a_{i+1}+a_{i}},
$$


or equivalently,

$$
\frac{b_{2 n+1-i}}{b_{i}} \leq \frac{b_{2 n-i}}{b_{i+1}}
$$

In light of (2.1), we see that $a_{n+1} \leq a_{n-1}$, and thus we have

$$
\frac{b_{n+1}}{b_{n}}=\frac{a_{n+1}+a_{n}}{a_{n}+a_{n-1}} \leq 1 .
$$

Next, we proceed to prove (2.4). Since $\frac{a_{0}}{a_{2 n-1}} \leq \frac{a_{1}}{a_{2 n-2}}$, we get that

$$
\frac{a_{0}}{a_{2 n-1}+a_{2 n}} \leq \frac{a_{1}+a_{0}}{a_{2 n-2}+a_{2 n-1}}
$$

that is,

$$
\frac{b_{0}}{b_{2 n}} \leq \frac{b_{1}}{b_{2 n-1}}
$$

For $2 \leq i \leq n-1$, in view of (2.2) we find that

$$
\frac{a_{i-2}}{a_{2 n-i+1}} \leq \frac{a_{i-1}}{a_{2 n-i}} \leq \frac{a_{i}}{a_{2 n-i-1}} .
$$

By Lemma 2.2, we have

$$
\frac{a_{i-1}+a_{i-2}}{a_{2 n-i+1}+a_{2 n-i}} \leq \frac{a_{i}+a_{i-1}}{a_{2 n-i}+a_{2 n-i-1}}
$$

which can be expressed as

$$
\frac{b_{i-1}}{b_{2 n-i+1}} \leq \frac{b_{i}}{b_{2 n-i}}
$$

From (2.2) it is clear that $a_{n-2} \leq a_{n+1}$ and $a_{n-1} \leq a_{n}$, and hence

$$
\frac{b_{n-1}}{b_{n+1}}=\frac{a_{n-1}+a_{n-2}}{a_{n+1}+a_{n}} \leq 1 .
$$

The case $m=2 n+1$ can be dealt with in the same manner. This completes the proof.

The third lemma is concerned with an inequality of increasing positive sequences.

Lemma 2.4 For any nondecreasing positive sequence $\left\{a_{k}\right\}_{0 \leq k \leq m}$, we have

$$
\frac{m(m+1)}{2} a_{m}^{2}+a_{m} a_{m-1} \geq\left(\sum_{k=0}^{m-2}(m-1-k) a_{k}\right) a_{m-1}+\left(\sum_{k=0}^{m} a_{k}\right) a_{m-2} .
$$


Proof. Since $0<a_{0} \leq a_{1} \leq \cdots \leq a_{m-1} \leq a_{m}$, we have

$$
\begin{gathered}
\frac{m(m+1)}{2} a_{m}^{2}+a_{m} a_{m-1}-\left(\sum_{k=0}^{m-2}(m-1-k) a_{k}\right) a_{m-1}-\left(\sum_{k=0}^{m} a_{k}\right) a_{m-2} \\
\geq \frac{m(m+1)}{2} a_{m}^{2}+a_{m} a_{m-1}-\sum_{k=0}^{m-2}(m-1-k) a_{m}^{2}-\sum_{k=1}^{m} a_{m}^{2}-a_{m} a_{m-1},
\end{gathered}
$$

which simplifies to zero, as desired.

Proof of Theorem 2.1. We use induction on the degree $m$ of $P(x)$. Let

$$
P(x)=\sum_{k=0}^{m} a_{k} x^{k}
$$

where $0<a_{0} \leq a_{1} \leq \cdots \leq a_{m-1} \leq a_{m}$.

When $m=2$, we have

$$
P(x+1)=a_{2} x^{2}+\left(a_{1}+2 a_{2}\right) x+a_{0}+a_{1}+a_{2} .
$$

Note that $a_{2} \leq a_{0}+a_{1}+a_{2}, a_{0}+a_{1}+a_{2} \leq a_{1}+2 a_{2}$. Therefore, the theorem holds for $m=2$.

Now assume that the theorem holds for polynomials of degree $m-1$. We need to show that it is also true for polynomials $P(x)$ of degree $m$. Suppose that

$$
P(x+1)=\sum_{k=0}^{m} a_{k}(x+1)^{k}=\sum_{k=0}^{m} d_{k} x^{k} .
$$

We wish to prove that

$$
\frac{d_{m}}{d_{0}} \leq \frac{d_{m-1}}{d_{1}} \leq \cdots \leq \frac{d_{m-i}}{d_{i}} \leq \cdots \leq \frac{d_{m-\left[\frac{m-1}{2}\right]}}{d_{\left[\frac{m-1}{2}\right]}} \leq 1
$$

and

$$
\frac{d_{0}}{d_{m-1}} \leq \frac{d_{1}}{d_{m-2}} \leq \cdots \leq \frac{d_{i-1}}{d_{m-i}} \leq \cdots \leq \frac{d_{\left[\frac{m}{2}\right]-1}}{d_{m-\left[\frac{m}{2}\right]}} \leq 1
$$

Let

$$
Q(x)=\sum_{k=0}^{m-1} a_{k+1} x^{k}
$$

Then

$$
P(x+1)=a_{0}+(x+1) Q(x+1) .
$$

By the induction hypothesis and Lemma 2.3, we deduce that the polynomial

$$
(x+1) Q(x+1)=d_{0}-a_{0}+\sum_{k=1}^{m} d_{k} x^{k}
$$


is ratio monotone. It follows that

$$
\frac{d_{m}}{d_{0}-a_{0}} \leq \frac{d_{m-1}}{d_{1}} \leq \cdots \leq \frac{d_{m-i}}{d_{i}} \leq \cdots \leq \frac{d_{m-\left[\frac{m-1}{2}\right]}}{d_{\left[\frac{m-1}{2}\right]}} \leq 1
$$

and

$$
\frac{d_{0}-a_{0}}{d_{m-1}} \leq \frac{d_{1}}{d_{m-2}} \leq \cdots \leq \frac{d_{i-1}}{d_{m-i}} \leq \cdots \leq \frac{d_{\left[\frac{m}{2}\right]-1}}{d_{m-\left[\frac{m}{2}\right]}} \leq 1 .
$$

Clearly, (2.6) follows from (2.8). To prove (2.7), it remains to show that

$$
\frac{d_{0}}{d_{m-1}} \leq \frac{d_{1}}{d_{m-2}}
$$

From (2.5), we see that

$$
d_{0}=\sum_{k=0}^{m} a_{k}, \quad d_{m-1}=a_{m-1}+m a_{m},
$$

and

$$
d_{1}=\sum_{k=0}^{m} k a_{k}, \quad d_{m-2}=a_{m-2}+(m-1) a_{m-1}+\left(\begin{array}{c}
m \\
2
\end{array}\right) a_{m} .
$$

Consequently, it suffices to show that

$$
\frac{\sum_{k=0}^{m} a_{k}}{a_{m-1}+m a_{m}} \leq \frac{\sum_{k=0}^{m} k a_{k}}{a_{m-2}+(m-1) a_{m-1}+\left(\begin{array}{c}
m \\
2
\end{array}\right) a_{m}},
$$

or equivalently,

$$
\begin{gathered}
\left(\sum_{k=0}^{m} k a_{k}\right) a_{m-1}+\left(\sum_{k=0}^{m} m k a_{k}\right) a_{m}-\left(\sum_{k=0}^{m} a_{k}\right) a_{m-2} \\
-\left(\sum_{k=0}^{m}(m-1) a_{k}\right) a_{m-1}-\left(\sum_{k=0}^{m}\left(\begin{array}{c}
m \\
2
\end{array}\right) a_{k}\right) a_{m} \geq 0 .
\end{gathered}
$$

The left hand side of the above inequality can be simplified to

$$
\left(\sum_{k=0}^{m} \frac{2 k-m+1}{2} a_{k}\right) m a_{m}+\left(\sum_{k=0}^{m}(k-m+1) a_{k}\right) a_{m-1}-\left(\sum_{k=0}^{m} a_{k}\right) a_{m-2},
$$

which can be rewritten as a sum of

$$
\left(\sum_{k=0}^{m-1} \frac{2 k-m+1}{2} a_{k}\right) m a_{m}
$$


and

$$
\frac{m(m+1)}{2} a_{m}^{2}+a_{m} a_{m-1}-\left(\sum_{k=0}^{m-2}(m-1-k) a_{k}\right) a_{m-1}-\left(\sum_{k=0}^{m} a_{k}\right) a_{m-2} .
$$

By Lemma 2.4, the sum in (2.11) is nonnegative. The sum in (2.10) is also nonnegative, since

$$
\begin{aligned}
& \sum_{k=0}^{m-1} \frac{2 k-m+1}{2} a_{k}=\sum_{k=\left[\frac{m-1}{2}\right]+1}^{m-1} \frac{2 k-m+1}{2} a_{k}-\sum_{k=0}^{\left[\frac{m-1}{2}\right]} \frac{m-1-2 k}{2} a_{k} \\
& =\sum_{k=0}^{m-2-\left[\frac{m-1}{2}\right]} \frac{m-1-2 k}{2} a_{m-1-k}-\sum_{k=0}^{\left[\frac{m-1}{2}\right]} \frac{m-1-2 k}{2} a_{k} \\
& =\sum_{k=0}^{\left[\frac{m-1}{2}\right]} \frac{m-1-2 k}{2}\left(a_{m-1-k}-a_{k}\right)
\end{aligned}
$$

which is nonnegative, and thus the proof is complete.

Theorem 2.1 leads to the following result of Llamas and Martínez-Bernal [9], since the ratio monotonicity implies log-concavity of $P(x+1)$ and the log-concavity of $P(x+1)$ implies the $\log$-concavity of $P(x+c)$ for $c \geq 1$ by a criterion of Brenti $[4,5]$.

Corollary 2.5 If $P(x)$ is a polynomial with nonnegative and nondecreasing coefficients, then for any $c \geq 1$ the polynomial $P(x+c)$ is log-concave and has no internal zero coefficients.

Theorem 2.1 also serves as a simple proof of the ratio monotonicity of the Boros-Moll polynomials $P_{m}(x)$, which were introduced by Boros and Moll [2] in their study of the following quartic integral

$$
\int_{0}^{+\infty} \frac{1}{\left(t^{4}+2 x t^{2}+1\right)^{m+1}} d t=\frac{\pi}{2^{m+3 / 2}(x+1)^{m+1 / 2}} P_{m}(x) .
$$

Let

$$
c_{k}(m)=2^{-2 m+k}\left(\begin{array}{c}
2 m-2 k \\
m-k
\end{array}\right)\left(\begin{array}{c}
m+k \\
k
\end{array}\right) .
$$

Boros and Moll showed that

$$
P_{m}(x)=\sum_{k=0}^{m} c_{k}(m)(x+1)^{k}
$$

They also observed that, for $0 \leq k \leq m-1$,

$$
\frac{c_{k}(m)}{c_{k+1}(m)}=\frac{(2 m-2 k-1)(k+1)}{(m-k)(m+k+1)}<1 \text {. }
$$


Thus, $P_{m}(x-1)$ is a polynomial with nonnegative and nondecreasing coefficients. Boros and Moll [2] proved that $P_{m}(x)$ is unimodal for any $m \geq 0$, and Moll [10] conjectured that $P_{m}(x)$ is $\log$-concave for any $m$. This conjecture was confirmed by Kauers and Paule [8]. The ratio monotonicity of $P_{m}(x)$ was established by Chen and Xia and the proof is quite involved and heavily depends on inequalities on the coefficients. The proof of Theorem 2.1 shows that the log-concavity and ratio monotonicity only depend on the nondecreasing property of the coefficients of $P_{m}(x-1)$.

Acknowledgments. This work was supported by the 973 Project, the PCSIRT Project of the Ministry of Education, and the National Science Foundation of China.

\section{References}

[1] J. Alvarez, M. Amadis, G. Boros, D. Karp, V.H. Moll and L. Rosales, An extension of a criterion for unimodality, Electron. J. Combin. 8 (2001), \#R30.

[2] G. Boros and V.H. Moll, A sequence of unimodal polynomials, J. Math. Anal. Appl. 237 (1999), 272-285.

[3] G. Boros and V.H. Moll. A criterion for unimodality, Electron. J. Combin. 6 (1999), \#R10.

[4] F. Brenti, Unimodal, log-concave, and Pólya frequency sequences in combinatorics, Mem. Amer. Math. Soc. 413 (1989), 1-106.

[5] F. Brenti, Log-concave and unimodal sequences in algebra, combinatorics and geometry: an update, Contemp. Math. 178 (1994), 71-89.

[6] W.Y.C. Chen and E.X.W. Xia, The ratio monotonicity of the $q$-derangement numbers, Discrete Math., to appear.

[7] W.Y.C. Chen and E.X.W. Xia, The ratio monotonicity of the Boros-Moll polynomials, Math. Comput. 78 (2009), 2269-2282.

[8] M. Kauers and P. Paule, A computer proof of Moll's log-concavity conjecture, Proc. Amer. Math. Soc. 135 (2007), 3847-3856.

[9] A. Llamas, J. Martínez-Bernal, Nested log-concavity, Commun. Algebra 38 (2010), 1968-1981.

[10] V.H. Moll, The evaluation of integrals: A personal story, Notices Amer. Math. Soc. 49 (2002), 311-317.

[11] R.P. Stanley, Log-concave and unimodal sequences in algebra, combinatorics and geometry, Ann. New York Acad. Sci 576 (1989), 500-535.

[12] Y. Wang and Y.-N. Yeh, Proof of a conjecture on unimodality, European J. Combin. 26 (2005), 617-627. 